\title{
光系统II实际光化学量子效率对光的响应模型的比较
}

\author{
叶子飘 $^{1^{*}}$ 胡文海 ${ }^{2}$ 间小红 ${ }^{2,3}$
}

${ }^{1}$ 井冈山大学数理学院, 江西吉安 343009 ; $^{2}$ 井冈山大学生命科学学院, 江西吉安 343009; ${ }^{3}$ 南京林业大学森林资源与环境学院, 南京 210037

摘 要 为了比较光系统II实际光化学量子效率 $\left(\Phi_{\mathrm{PSIII}}\right)$ 对光的响应机理模型(简称机理模型)、负指数模型和指数模型的优缺 点, 用LI-6400-40B光合作用测定仪控制 $\mathrm{CO}_{2}$ 浓度和温度, 测量了剑叶金鸡菊(Coreopsis lanceolata)、黄荆(Vitex negundo)和大 狼杷草(Bidens frondosa)的电子传递速率 $(E T R)$ 对光的响应曲线 $(E T R-I)$ 和 $\Phi_{\mathrm{PSII}}$ 对光的响应曲线 $\left(\Phi_{\mathrm{PSII}}-I\right)$, 然后用这 3 个模型分别 拟合了这些数据。拟合结果表明: 3 个模型都可以较好地拟合这 3 种植物的ETR-I的响应数据和 $\Phi_{\mathrm{PSII}}-I$ 的响应数据, 但由指数模 型拟合ETR-I和 $\Phi_{\mathrm{PSII}}-I$ 的响应数据得到相应的饱和光强 $\left(P A R_{\mathrm{sat}}\right)$ 和光系统II最大光能利用效率 $\left(F_{\mathrm{v}} / F_{\mathrm{m}}\right)$ 之间存在显著差异, 且估 算的饱和光强远低于实测值。由机理模型可知, $\Phi_{\mathrm{PSII}}$ 不仅与光强的函数有关, 还与植物的内禀特性有关, 即与天线色素分子的 本征光能吸收截面、激子的传递效率、能级的简并度、光化学反应常数、热耗散常数和处于最低激发态的平均寿命等参数有 关。此外, 由机理模型还可知, $\Phi_{\mathrm{PSII}}$ 随光强的增加而下降的原因是捕光色素分子的有效光能吸收截面随光强增加而降低。 关键词＼cjkstart光系统II; 实际光化学量子效率; 电子传递速率; 机理模型; 天线色素分子

引用格式: 叶子飘, 胡文海, 间小红 (2016). 光系统II实际光化学量子效率对光的响应模型的比较. 植物生态学报, 40, 1208-1217. doi: 10.17521/cjpe.2015.0470

\section{Comparison on light-response models of actual photochemical efficiency in photosystem II}

YE Zi-Piao ${ }^{1 *}$, HU Wen-Hai ${ }^{2}$, and YAN Xiao-Hong ${ }^{2,3}$

${ }^{1}$ Maths \& Physics College, Jinggangshan University, Ji'an, Jiangxi 343009, China; ${ }^{2}$ School of Life Sciences, Jinggangshan University, Ji'an, Jiangxi 343009, China; and ${ }^{3}$ College of Forest Resources and Environment, Nanjing Forestry University, Nanjing 210037, China

\section{Abstract}

Aims The objective of this study was to compare the merits and demerits of three models (i.e., a mechanistic model, a negative exponential model and an exponential model) to simulate the light-response curves of actual photochemical efficiency ( $\left.\Phi_{\mathrm{PSII}}-I\right)$. Moreover, it was to reveal the mechanism that $\Phi_{\mathrm{PSII}}$ decreased with light intensity increasing.

Methods The electron transport rate $(E T R)$ and the $\Phi_{\mathrm{PSII}}$ of Coreopsis lanceolata, Vitex negundo and Bidens frondosa were measured by LI-6400-40B under controlled $\mathrm{CO}_{2}$ concentrations and temperatures, then light-response curves of $E T R-I$ and $\Phi_{\mathrm{PSII}}-I$ were simulated by a mechanistic model, a negative exponential model and an exponential model, respectively.

Important findings The fitted results showed that $E T R-I$ and $\Phi_{\mathrm{PSII}}-I$ data of the three plants fit well to the three models. However, the saturation light intensity $\left(P A R_{\mathrm{sat}}\right)$ and maximum efficiency of photosystem II $\left(F_{\mathrm{v}} / F_{\mathrm{m}}\right)$ estimated by exponential model for ETR-I and $\Phi_{\mathrm{PSII}}-I$ were greatly different from the measured data. Moreover, the mechanistic model revealed that the $\Phi_{\text {PSII }}$ for the three species decreased with increasing $I$ as the effective light energy absorption cross-section of light-harvesting pigments decreased. At the same time, it showed that $\Phi_{\text {PSII }}$ depended not only on $I$, but also on eigen-absorption cross-section of light-harvesting pigment, efficiency of exciton, photochemical constant, heat dissipation constant and average lifetime of light-harvesting pigment-protein complex in the lowest state.

Key words photosystem II; actual photochemical efficiency; electron transport rate; a mechanistic model; lightharvesting pigment-protein complex

Citation: Ye ZP, Hu WH, Yan XH (2016). Comparison on light-response models of actual photochemical efficiency in photosystem II. Chinese Journal of Plant Ecology, 40, 1208-1217. doi: 10.17521/cjpe.2015.0470

收稿日期Received: 2015-12-22 接受日期Accepted: 2016-07-19

*E-mail: yezp@jgsu.edu.cn 
光系统II (PSII)的实际光化学量子效率 $\left(\Phi_{\mathrm{PSII}}\right)$ 为 PSII反应中心部分关闭时的实际原初光能捕获效 率, 表征作用光下PSII吸收光能用于原初醌电子受 体 $\mathrm{Q}_{\mathrm{A}}$ 还原的实际运行效率, 是一个被广泛应用的荧 光参数。由于它与通过PSII反应中心的线性电子传递 $(L E T)$ 有直接的联系, 可快速地确定光强变化时或其 他环境条件下 PSII 反应中心的作用效率 (Baker, 2008), 所以, 它又被称为 PSII 的运行效率(PSII operating efficiency)。因此, 研究它的变化规律具有 重要的理论价值和实际意义(Ritchie \& Bunthawin, 2010; Takahashi \& Badger, 2011; Silsbe \& Kromkamp, 2012; Niyogi \& Truong, 2013; 安东升等, 2015)。

按照Genty等(1989)关于电子传递速率(ETR)或 光合电子流 $(J)$ 的理论, $E T R$ 的大小可以由公式 $E T R=$ $\alpha^{\prime} \times \beta^{\prime} \times \Phi_{\mathrm{PSII}} \times I$ 得到, 式中的 $\Phi_{\mathrm{PSII}}$ 就是PSII的实际 光化学量子效率, $\alpha^{\prime} 、 \beta^{\prime} 、 I$ 分别为光能在PSII和PSI 的分配系数(无量纲)、叶片的光吸收系数(无量纲) 和光强。而 $\Phi_{\mathrm{PSII}}=\left(F_{\mathrm{m}}^{\prime}-F_{\mathrm{s}}\right) / F_{\mathrm{m}}^{\prime}$ (Genty et al., 1989; Krall \& Edward, 1992), 其中 $F^{\prime}{ }_{m}$ 为光下的最大荧光, $F_{\mathrm{s}}$ 为光下稳态苂光。实验结果表明: $\Phi_{\mathrm{PSII}}$ 是一个依赖 光强的函数, 它随光强的增加而下降(Genty et al., 1989; Krall \& Edward, 1992; Ritchie, 2008; Ritchie \& Bunthawin, 2010; Silsbe \& Kromkamp, 2012; 冯汉青 等, 2014; 安东升等, 2015; 林琭等, 2015; Ware et $a l ., 2015)$ 。同时, $\Phi_{\mathrm{PSII}}$ 也可以由几个经验模型直接拟 合(Laws et al., 2002; Smyth et al., 2004; Ritchie, 2008; Ritchie \& Bunthawin, 2010; Silsbe \& Kromkamp, 2012), 但这些经验模型主要应用于藻类和浮 游植物(Laws et al., 2002; Smyth et al., 2004; Silsbe \& Kromkamp, 2012)和少数几种陆生植物(Ritchie, 2008; Ritchie \& Bunthawin, 2010), 其他陆生植物应 用较少。已有的研究结果表明: 由指数模型分别拟 合 $E T R$ 对光的响应曲线 $(E T R-I)$ 和 $\Phi_{\mathrm{PSII}}$ 对光的响应曲 线 $\left(\Phi_{\mathrm{PSII}}-I\right)$ 的响应数据得到相应的饱和光强 $\left(P A R_{\mathrm{sat}}\right)$ 和植物经过暗适应的PSII最大光能利用效率 $F_{\mathrm{v}} / F_{\mathrm{m}}$ ( $F_{\mathrm{v}}$ 为可变苂光, $F_{\mathrm{m}}$ 为最大荧光产量) 差异较大, 且所 估算的饱和光强与实际测量值差异较大(Laws et al., 2002; Smyth et al., 2004; Silsbe \& Kromkamp, 2012); 负指数模型拟合 $E T R-I$ 和 $\Phi_{\mathrm{PSII}}-I$ 的响应数据得到的 饱和光强与实测值符合, 但由此模型得到光强为 0 时的最大光化学量子效率 $\left(\Phi_{\mathrm{PSIImax}}\right)$ 之间存在较大的 差异(Ritchie, 2008)。由此可知, 由负指数和指数模
型拟合 ETR-I 和 $\Phi_{\mathrm{PSII}}-I$ 的响应曲线分别得到的 $\Phi_{\mathrm{PSIImax}}$ 之间和 $F_{\mathrm{v}} / F_{\mathrm{m}}$ 之间存在明显差异, 但尚不清 楚产生这种现象的原因。此外, 这两个模型也不能 解释为什么植物的 $\Phi_{\mathrm{PSII}}$ 会随光强的增加而下降。 正是由于存在这两个问题, 所以限制了这些模型的 应用。

而由 $\Phi_{\mathrm{PSII}}=\left(F_{\mathrm{m}}^{\prime}-F_{\mathrm{s}}\right) / F_{\mathrm{m}}^{\prime}$ 的定义可知, $\Phi_{\mathrm{PSII}}$ 与光 合作用原初反应中的PSII的开闭和天线色素分子发 射的荧光过程紧密相连。首先, 天线色素分子吸收 光能后将由基态跃迁到高能态, 然后这些激发能将 分配到光化学反应、热耗散和发射叶绿素苂光这3 条主要途径且它们彼此竞争 (Oxborough, 2004; Baker, 2008), 这种分配过程直接影响到随后的光合 电子传递与同化力的形成。而原初反应是一个纯粹 的物理过程, 涉及天线色素分子的光能吸收、量子 态的改变、激子的共振传递和退激发等, 这些过程 又是由天线色素分子的内禀特性所决定的 (Govindjee, 2002; Baker, 2008; Richter et al., 2008; Panitchayangkoon et al., 2010; Sarovar et al., 2010)。 此外, 光合作用也与光合能力的形成 (如 ATP 和 NADPH的形成) (Govindjee, 2002; Nelson \& Yocum, 2006; Ye et al., 2013a, 2013b)和与碳同化相连的酶 动力过程有关(Lin et al., 2015)。由此推测: (1) $\Phi_{\mathrm{PSII}}$ 的大小应该与植物的天线色素分子、PSII的内禀特 性和酶动力学过程密切相关; (2) $\Phi_{\mathrm{PSII}}$ 随光强的增加 而下降可能与捕光色素分子的有效光能有关; (3)由 负指数和指数模型拟合 $E T R-I$ 和 $\Phi_{\mathrm{PSII}}-I$ 的响应曲线 分别得到的 $\Phi_{\mathrm{PSIImax}}$ 之间和 $F_{\mathrm{v}} / F_{\mathrm{m}}$ 之间明显存在差异 可能是由于LI-6400-40B苂光仪设定的叶片光能吸 收系数为 0.84 和光能在PSII和PSI之间的分配比例为 0.5 并非适合所有的植物。

针对以上的 3 个推测, 在本研究中, 首先, 我们 基于光合电子流对光的响应机理模型(Ye et al., 2013a, 2013b)推导出 $\Phi_{\mathrm{PSII}}$ 对光的响应模型, 确定其 变化规律与PSII天线色素分子的内禀特性之间的关 系; 其次, 利用 $\Phi_{\mathrm{PSII}}$ 对光响应的机理模型、负指数模 型和指数模型分别拟合剑叶金鸡菊 (Coreopsis lanceolata)、黄荆(Vitex negundo)和大狼杷草(Bidens frondosa) 在正常条件下 $\Phi_{\mathrm{PSII}}$ 对光的响应曲线, 判断 所推导的 $\Phi_{\mathrm{PSII}}$ 对光的响应模型是否合理和有效, 并 与负指数模型和指数模型的拟合结果进行比较, 以 确定这些模型的优缺点; 再次, 用所测量的ETR对 
光的响应数据验证我们的推测, 即植物的 $\Phi_{\mathrm{PSII}}$ 随光 强的增加而下降可能与其天线色素分子的有效光能 吸收截面特性密切相关; 最后, 利用所拟合的结果 确定LI-6400-40B苂光仪设定的叶片光能吸收系数 为 0.84 和光能在PSII和PSI的分配比例为 0.5 是否适 合剑叶金鸡菊、黄荆和大狼杷草。因此, 这些问题 的探讨将为研究植物的 $\Phi_{\mathrm{PSII}}$ 对光的响应以及 $\Phi_{\mathrm{PSII}}$ 随 光强的增加而下降的机理提供理论基础, 同时也为 研究植物的ETR-I和 $\Phi_{\mathrm{PSII}}-I$ 提供数学工具。

\section{1 材料和方法}

\section{1 材料}

供试材料为自然生长在江西省吉安市井冈山大 学校园内的剑叶金鸡菊、黄荆和大狼杷草。

\section{2 测定及计算方法}

在2013年6月连续的几个晴天内, 在9:00-11:30 和14:30-16:30, 随机选5株长势较为一致的健壮植 株用LI-6400-40B (LI-COR, Lincoln, USA)测定其 $E T R$ 对光的响应曲线和其他参数。在测定过程中, 气室 $\mathrm{CO}_{2}$ 浓度控制为 $(390 \pm 2) \mu \mathrm{mol} \cdot \mathrm{mol}^{-1}$, 气室温 度设置为 $(35 \pm 1){ }^{\circ} \mathrm{C}$, 气室的空气相对湿度设置为 $50 \%-70 \%$ 。光合有效辐射 $(P A R)$ 设置为2 000、1800、 $1600 、 1400 、 1200 、 1000 、 800 、 600 、 400 、 200 、$ 100、 50 和 $0 \mu \mathrm{mol} \cdot \mathrm{m}^{-2} \cdot \mathrm{s}^{-1}$ 共 13 个水平, 并分别于各光 强下测定剑叶金鸡菊、黄荆和大狼杷草的光合电子 流 $(J)$ 或ETR 和其他参数, 每个测定点的响应时间在 $120 \mathrm{~s}$ 以上。然后分别用植物光合电子流对光响应的 机理模型、负指数模型和指数模型拟合这 3 种植物的 光合电子流对光的响应曲线, 得到其光响应曲线的 初始斜率 $(\alpha)$ 或光强为 0 时的最大光化学量子效率 $\left(\Phi_{\mathrm{PSIImax}}\right)$ 或植物经过暗适应的PSII最大光化学量子 产量 $\left(F_{\mathrm{v}} / F_{\mathrm{m}}\right)$ 、饱和光强 $\left(P A R_{\mathrm{sat}}\right)$ 、最大光合电子流 $\left(J_{\max }\right)$ 或最大电子传递速率 $\left(E T R_{\max }\right)$, 并用相关的参 数估算天线色素分子的有效光能吸收截面对光的响 应曲线等。

\section{3 机理模型的推导}

植物叶片的天线色素分子吸收的光能将在光化 学反应、热耗散和荧光发射等3条途径上竞争分配 (Oxborough, 2004; Baker, 2008), Ye等(2013a, 2013b) 在此基础上构建了一个ETR对光的响应机理模型。 其数学表达式为:

$$
E T R=\frac{\alpha^{\prime} \beta^{\prime} N_{0} \sigma_{\mathrm{ik}} \varphi}{S} \times \frac{1-\frac{\left(1-g_{\mathrm{i}} / g_{\mathrm{k}}\right) \sigma_{\mathrm{ik}} \tau}{\xi_{3}+\left(\xi_{1} k_{\mathrm{p}}+\xi_{2} k_{\mathrm{D}}\right) \tau} I}{1+\frac{\left(1+g_{\mathrm{i}} / g_{\mathrm{k}}\right) \sigma_{\mathrm{ik}} \tau}{\xi_{3}+\left(\xi_{1} k_{\mathrm{p}}+\xi_{2} k_{\mathrm{D}}\right) \tau} I} I
$$

式中的符号如 $\varphi 、 N_{0} 、 S 、 g_{\mathrm{i}} 、 g_{\mathrm{k}} 、 k_{\mathrm{P}} 、 k_{\mathrm{D}} 、 \xi_{1} 、 \xi_{2} 、 \xi_{3} 、$ $\sigma_{\mathrm{ik}}$ 和 $\tau$ 等皆为描述天线色素分子物理特性的参数, 详见相关文献(Ye et al., 2013a, 2013b; 叶子飘等, 2014), $\alpha^{\prime}$ 为叶片的光吸收系数, $\beta^{\prime}$ 为光能在 PSII和 PSI两个光系统中的分配比例。其中 $\alpha^{\prime}$ 一般取值为 0.84 (Ehleringer \& Pearcy, 1983; Krall \& Edwards, 1992), $\beta^{\prime}$ 一般取值为0.5 (Ehleringer \& Pearcy, 1983; Krall \& Edwards, 1992; Major \& Dunton, 2002)。

由于在一定的环境下植物叶片天线色素分子的 物理参数是确定的, 可以假设: $\alpha=\alpha^{\prime} \beta^{\prime} N_{0} \varphi \sigma_{\mathrm{ik}} / S$ $\left(\mu \mathrm{mol}\right.$ electrons $\left.\cdot(\mu \mathrm{mol} \text { photons })^{-1}\right), \beta=\left(\left(1-g_{\mathrm{i}} / g_{\mathrm{k}}\right) \sigma_{\mathrm{ik}} \tau\right) /$ $\left(\xi_{3}+\left(\xi_{1} k_{\mathrm{P}}+\xi_{2} k_{\mathrm{D}}\right) \tau\right)\left(\mathrm{m}^{2} \cdot \mathrm{s} \cdot(\mu \mathrm{mol} \text { photons })^{-1}\right), \gamma=((1+$ $\left.\left.g_{\mathrm{i}} / g_{\mathrm{k}}\right) \sigma_{\mathrm{ik}} \tau\right) /\left(\xi_{3}+\left(\xi_{1} k_{\mathrm{P}}+\xi_{2} k_{\mathrm{D}}\right) \tau\right)\left(\mathrm{m}^{2} \cdot \mathrm{s} \cdot(\mu \text { mol photons })^{-1}\right)$, 那么公式(1)可以简化为:

$$
E T R=\alpha \frac{1-\beta I}{1+\gamma I} I
$$

由公式(2)可以得到植物叶片的饱和光强 $\left(P A R_{\mathrm{sat}}\right)$ 和 最大电子传递速率 $\left(E T R_{\text {max }}\right)$ 分别为:

$$
P A R_{\mathrm{sat}}=\frac{\sqrt{(\beta+\gamma) / \beta}-1}{\gamma} \text { 和 } E T R_{\max }=\alpha\left(\frac{\sqrt{\beta+\gamma}-\sqrt{\beta}}{\gamma}\right)^{2}
$$

此外, 按照Krall和Edward (1992)给出的光合电 子流对光的响应公式:

$$
E T R=\alpha^{\prime} \beta^{\prime} \Phi_{\mathrm{PSII}} I
$$

对比公式(1)和公式(3), 可以得到 $\Phi_{\mathrm{PSII}}$ 与光强 之间的关系式为:

$$
\Phi_{\mathrm{PSII}}=\frac{N_{0} \sigma_{\mathrm{ik}} \varphi}{S} \times \frac{1-\frac{\left(1-g_{\mathrm{i}} / g_{\mathrm{k}}\right) \sigma_{\mathrm{ik}} \tau}{\xi_{3}+\left(\xi_{1} k_{\mathrm{p}}+\xi_{2} k_{\mathrm{D}}\right) \tau} I}{1+\frac{\left(1+g_{\mathrm{i}} / g_{\mathrm{k}}\right) \sigma_{\mathrm{ik}} \tau}{\xi_{3}+\left(\xi_{1} k_{\mathrm{p}}+\xi_{2} k_{\mathrm{D}}\right) \tau} I}
$$

由公式(4)可知, $\Phi_{\mathrm{PSII}}$ 不仅与光强存在非线性关系, 还与天线色素分子的本征光能吸收截面 $\left(\sigma_{\mathrm{ik}}\right)$ 、天线 色素分子数 $\left(N_{0}\right)$ 、激子的传递效率 $(\varphi)$ 、激子处于最 低激发态的平均寿命 $(\tau)$ 、光化学反应速率 $\left(k_{\mathrm{P}}\right)$ 和热耗 散速率 $\left(k_{\mathrm{D}}\right)$ 、天线色素分子处于基态 $i$ 的简并度 $\left(g_{\mathrm{i}}\right)$ 和激发态 $k$ 的简并度 $\left(g_{\mathrm{k}}\right)$ 等参数存在密切的关系。

假 设 $\omega=N_{0} \varphi \sigma_{\mathrm{ik}} / S$ ( $\mu$ mol electrons $\cdot(\mu \mathrm{mol}$ photons) $)^{-1}$ ), 那么公式(4)可以简化为: 


$$
\Phi_{\mathrm{PSII}}=\omega \frac{1-\beta I}{1+\gamma I}
$$

这里的 $\omega$ 是 $\Phi_{\mathrm{PSII}}-I$ 曲线的初始斜率。由公式(5)可知, $\Phi_{\mathrm{PSII}}$ 随光强的增加而非线性下降。对比公式(2)和公 式(5)可知, $\alpha / \omega=\alpha^{\prime} \beta^{\prime}$, 由此也可以确定Dual-PAM100 所推荐的 $\alpha^{\prime}$ 和 $\beta^{\prime}$ 的值分别为 0.84 和 0.5 是否合理。

由公式(1)还我们还可以得到天线色素分子有 效光能吸收截面 $\left(\sigma_{\mathrm{ik}}^{\prime}\right)$ 与光强之间的关系(Ye et al., 2013a, 2013b)为:

$$
\sigma_{\mathrm{ik}}^{\prime}=\frac{\sigma_{\mathrm{ik}}}{1+\frac{\left(1+g_{\mathrm{i}} / g_{\mathrm{k}}\right) \sigma_{\mathrm{ik}} \tau I}{\xi_{3}+\left(\xi_{1} k_{P}+\xi_{2} k_{D}\right) \tau}}\left[1-\frac{\left(1-g_{\mathrm{i}} / g_{\mathrm{k}}\right) \sigma_{\mathrm{ik}} \tau I}{\xi_{3}+\left(\xi_{1} k_{P}+\xi_{2} k_{D}\right) \tau}\right]
$$

由公式(6)可知, $\sigma_{\mathrm{ik}}^{\prime}$ 随光强的增加呈非线性下降。对 比公式(6)和公式(4)可知, 对于一定植物在一定环 境条件下, $\Phi_{\mathrm{PSII}}$ 与 $\sigma_{\mathrm{ik}}^{\prime}$ 成正比, 即:

$$
\Phi_{\mathrm{PSII}}=N_{0} \varphi \sigma_{\mathrm{ik}}^{\prime} / S
$$

\section{4 经验模型的介绍}

现有拟合 $\Phi_{\mathrm{PSII}}-I$ 曲线的经验模型有负指数模型 (Ritchie, 2008; Ritchie et al., 2010)、指数模型(Webb et al., 1974; Smyth et al., 2004; Silsbe \& Kromkamp, 2012)和正切函数模型(Webb et al., 1974; Smyth et al., 2004), 其中以负指数模型和指数模型应用最为 广泛。负指数模型(Ritchie, 2008; Ritchie et al., 2010) 的数学表达式是:

$$
\Phi_{\text {PSII }}=\Phi_{\text {PSIImax }} \mathrm{e}^{-k_{w} I}
$$

式中的 $\Phi_{\mathrm{PSII} m a x}$ 是光强为 0 时的最大光化学量子效率, $k_{\mathrm{w}}$ 是一个常数, $I$ 为光强。与公式(8)相对应的ETR对 光的响应模型的数学表达式为:

$$
E T R=\alpha^{\prime} \beta^{\prime} I \Phi_{\text {PSIImax }} \mathrm{e}^{-k_{w} I}
$$

由公式(9)可以得到植物叶片的饱和光强和最 大电子传递速率分别为: $P A R_{\mathrm{sat}}=1 / k_{\mathrm{w}}$ 和 $E T R_{\text {max }}$ $=\alpha^{\prime} \beta^{\prime} P A R_{\text {sat }} \Phi_{\text {PSIImax }} \mathrm{e}^{-1}$ 。

指数模型(Webb et al., 1974; Smyth et al., 2004; Silsbe \& Kromkamp, 2012)的数学表达式是:

$$
\Phi_{\mathrm{PSII}}=\frac{F_{\mathrm{v}}}{F_{\mathrm{m}}} \times \frac{P A R_{\mathrm{sat}}}{I}\left[1-\exp \left(-I / P A R_{\mathrm{sat}}\right)\right]
$$

式中的 $P A R_{\mathrm{sat}} 、 F_{\mathrm{v}} / F_{\mathrm{m}}$ 和 $I$ 与以上的定义相同。由公式 $(10)$ 可以直接估算出植物叶片的饱和光强 $\left(P A R_{\mathrm{sat}}\right)$ 。与 公式(10)相对应的光合电子流对光的响应模型的数 学表达式为:
$E T R=\alpha^{\prime} \beta^{\prime}\left(F_{\mathrm{v}} / F_{\mathrm{m}}\right) P A R_{\text {sat }}\left[1-\exp \left(-I / P A R_{\mathrm{sat}}\right)\right]$

此外, 由公式(11)也可以直接估算植物叶片的 $P A R_{\text {sat }}$, 最大电子传递速率则为: $E T R_{\text {max }}=\alpha^{\prime} \beta^{\prime}\left(F_{\mathrm{v}} / F_{\mathrm{m}}\right)$ $P A R_{\text {sat }}[1-\exp (-1)]$ 。

\section{5 数据处理}

$E T R-I$ 曲线和 $\Phi_{\mathrm{PSII}}-I$ 曲线的实验数据处理用 SPSS 12.5; 用 Origin 7.0 作图, 然后用 Adobe Illustrator CS5进行图形处理, 采用SPSS 12.5作方差 差异显著性分析、ANOVA检验和配对样本 $t$ 检验, 用DPS 7.05软件作模型分析。本文将用AIC信息准 则并结合确定系数 $\left(R^{2}\right)$ 对 3 个模型的拟合结果进行 比较, 并判断 3 个模型的优缺点。其中AIC的计算参 考Akaike (1973)的方法, $R^{2}$ 则是直接由SPSS 12.5在 拟合数据后给出。AIC值越小, 说明拟合的模型既精 度高又简洁; $R^{2}$ 是衡量所建立模型效果好坏的指标 之一, 其值越大表明模型拟合得越好。此外, 为了比 较 $\alpha / \omega$ 与0.42 $\left(\alpha^{\prime} \beta^{\prime}=0.42\right)$ 之间是否存在显著性差异, 本文对此进行了配对样本 $t$ 检验，显著性水平设定 为 $\alpha=0.05$ 。文中的图和表中的部分数据均用平均值 土标准误差表示。

\section{6 叶绿素含量的测定}

取测定完光合电子流和净光合速率对光的响应 曲线的成熟叶片, 在不同叶片上取直径 $0.7 \mathrm{~cm}$ 的叶 圆片 30 片, 分3组, 分别浸入 $10 \mathrm{~mL} 80 \%$ 丙酮的提取 液中, 盖紧样品瓶盖并密封, 避光低温放置48 h至 叶圆片无色。然后参照Arnon (1949)的方法测量叶绿 素的含量, 重复5次取平均值。

\section{2 实验结果和分析}

\subsection{3 种植物叶片的电子传递速率对光的响应曲线 (ETR-I响应曲线)}

植物的光合电子流对光的响应曲线一般可以分 为3个阶段(Ralph \& Gademann, 2005): 第一阶段是 光合电子流随光强的增加而几乎线性增加; 第二阶 段是光合电子流随光强的增加缓慢增加到一个平 台; 第三阶段是光合电子流随光强的增加而下降, 出现光PSII的动力学下调(每个光强点的测量时间 小于60 s)或光抑制现象(每个光强点的测量时间大 于180 s)。由图1可知, 剑叶金鸡菊和大狼杷草的光 合电子流随光强的增加而下降, 而黄荆的光合电子 流随光强的增加一直在增加，尚未出现下降。

由图1和表1可知, 由公式(2)和(9)得到剑叶金 


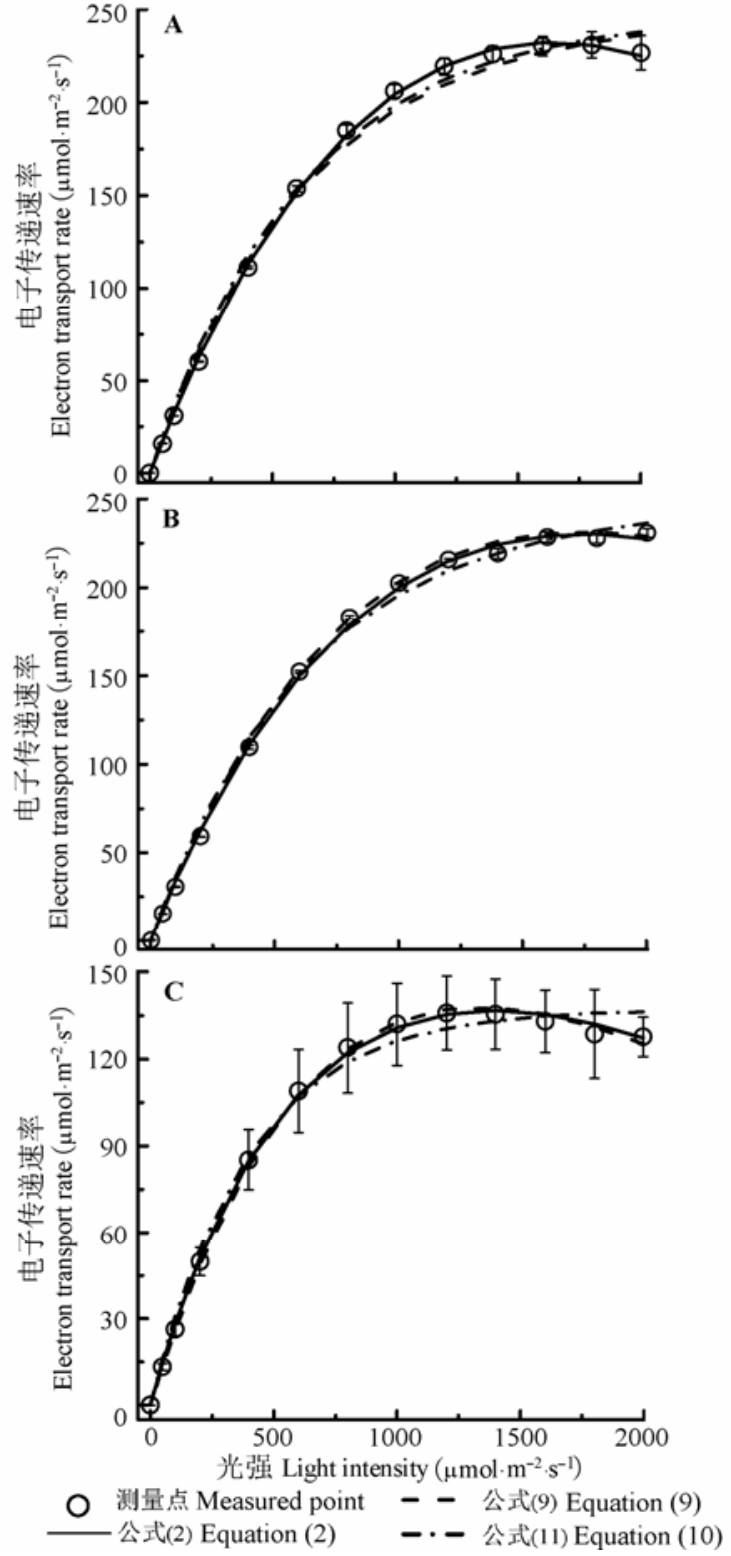

图1 剑叶金鸡菊(A)、黄荆 $(B)$ 和大狼杷草 $(C)$ 的电子传递速 率对光的响应曲线 $(E T R-I)$ (平均值土标准误差)。公式(2)为 $E T R=\alpha(1-\beta I) /(1+\gamma I) I$, 其中 $\alpha$ 为 $E T R-I$ 的初始斜率, $\beta$ 为光 抑制项, $\gamma$ 为光饱和项, $I$ 为光强; 公式(9)为 $E T R=\alpha^{\prime} \beta^{\prime} I \Phi_{\mathrm{PSIImax}}$ $\mathrm{e}^{-k_{w} I}$, 其中 $\alpha^{\prime}$ 为叶片光能吸收系数, $\beta^{\prime}$ 为光能在 2 个光系统的 分配比例, $\Phi_{\mathrm{PSIImax}}$ 为光强为 0 时的最大光化学量子效率, $k_{\mathrm{w}}$ 是 一个常数; 公式 (11) 为 $E T R=\alpha^{\prime} \beta^{\prime}\left(F_{\mathrm{v}} / F_{\mathrm{m}}\right) P A R_{\text {sat }}[1-\exp$ $\left.\left(-I / P A R_{\mathrm{sat}}\right)\right]$, 其中 $F_{\mathrm{v}} / F_{\mathrm{m}}$ 为光系统II的最大光量子效率, $P A R_{\text {sat }}$ 为饱和光强。

Fig. 1 Light-response curves of electron transport rate (ETRI) for Coreopsis lanceolata (A), Vitex negundo (B) and Bidens frondosa $(\mathbf{C})$ (mean $\pm S E)$. Equation (2) is ETR $=\alpha(1-\beta I) /(1+$ $\gamma I$ ) $I$. Here $\alpha$ is the initial slope of ETR-I, $\beta$ is the photoinhibition term, $\gamma$ is the saturation term, $I$ is light intensity; Equation (9) is $E T R=\alpha^{\prime} \beta^{\prime} I \Phi_{\text {PSIImax }} \mathrm{e}^{-k_{w} I}$. Here $\alpha^{\prime}$ is the light absorption coefficient of leaf, $\beta^{\prime}$ is the light energy distribution coefficient between photosystem II and photosystem I, $\Phi_{\text {PSIImax }}$ is the maximum photochemical quantum efficiency while $I=0, k_{\mathrm{w}}$ is a constant; Equation (11) is $E T R=\alpha^{\prime} \beta^{\prime}\left(F_{\mathrm{v}} / F_{\mathrm{m}}\right) P A R_{\text {sat }}\left[1-\exp \left(-I / P A R_{\text {sat }}\right)\right]$. Here $F_{\mathrm{v}} / F_{\mathrm{m}}$ is the maximum quantum of photosystem II, $P A R_{\text {sat }}$ is the saturation irradiance.
鸡菊、黄荆和大狼杷草的最大光合电子流和饱和光 强与实测值高度符合，而由公式(11)得到的 $E T R_{\text {max }}$ 分别为156.5、155.99和 $86.67 \mu \mathrm{mol} \cdot \mathrm{m}^{-2} \cdot \mathrm{s}^{-1}$, 测量值则 分别约为 $230.4 、 228.8$ 和 $135.8 \mu \mathrm{mol} \cdot \mathrm{m}^{-2} \cdot \mathrm{s}^{-1}$, 估算值 分别约为实测值的 $67.8 \%$ 、68.2\%和63.8\%; 由公式 (11) 得到的 $P A R_{\text {sat }}$ 分别为 $617.83 、 633.1$ 和 395.2 $\mu \mathrm{mol} \cdot \mathrm{m}^{-2} \cdot \mathrm{s}^{-1}$, 测量值分别约为 $1600 、 1750$ 和 1400 $\mu \mathrm{mol} \cdot \mathrm{m}^{-2} \cdot \mathrm{s}^{-1}$, 估算值尚未达到实测值的一半 $($ 表 1$)$ 。 此外, 由表1的数据可知, 公式(2)、(9)和(11)拟合3 种植物的光合电子流对光的响应曲线的 $R^{2}$ 都在 0.994 以上。如果用确定系数的大小表示模型的拟合 与实际测量曲线的符合度, 那么(2)式的拟合效果最 好, (11)式的拟合效果最差。如果以AIC值的大小作 为衡量标准, 则以公式(9)的拟合效果最好, 公式 (11)的拟合效果最差(表1)。

\section{2 三种植物叶片的PSII实际光化学量子效率对} 光的响应曲线 $\left(\Phi_{\mathrm{PSII}}-\boldsymbol{I}\right)$

由图2可知, 3 种植物的 $\Phi_{\mathrm{PSII}}$ 随光强的增加而非 线性下降, 且剑叶金鸡菊和黄荆的下降速度略有差 异, 但大狼杷草的 $\Phi_{\mathrm{PSII}}$ 随光强的增加而下降的速度 最快。由图2还可知, 3 个模型都能较好地拟合剑叶金 鸡菊、黄荆和大狼杷草的 $\Phi_{\mathrm{PSII}}-I$ 曲线, $R^{2}$ 超过 0.984 。 此外, 由表 2 的数据可知, 如果仅从 $R^{2}$ 值的大小衡量 3 个模型的优劣, 那么以机理模型的拟合效果最好, 指数模型最差; 如果以AIC值的大小衡量, 那么以 负指数模型的拟合效果最好, 拟合效果最差的是指 数模型。

除了可用公式(9)和(11)拟合ETR-I曲线分别得 到这3种植物的 $\Phi_{\mathrm{PSIImax}}$ 和 $F_{\mathrm{v}} / F_{\mathrm{m}}$ 的值外, 也可以由公 式 (8) 和 (10) 拟合 $\Phi_{\mathrm{PSII}}-I$ 曲线得到这 3 种植物的 $\Phi_{\mathrm{PSIImax}}$ 和 $F_{\mathrm{v}} / F_{\mathrm{m}}$ 的值(表2)。此外, 由公式(11)和(10) 分别拟合 $E T R-I$ 曲线和 $\Phi_{\mathrm{PSII}}-I$ 曲线得到的 $P A R_{\mathrm{sat}}$ 有较 大的差异。比如, 由公式(11)拟合剑叶金鸡菊的 $E T R-I$ 曲线得到的 $P A R_{\mathrm{sat}}$ 为 $617.83 \mu \mathrm{mol} \cdot \mathrm{m}^{-2} \cdot \mathrm{s}^{-1}$, 而由 公式(10)拟合剑叶金鸡菊的 $\Phi_{\mathrm{PSII}}-I$ 曲线得到的 $P A R_{\mathrm{sat}}$ 为 $824.95 \mu \mathrm{mol} \cdot \mathrm{m}^{-2} \cdot \mathrm{s}^{-1}$, 后者是前者的 1.33 倍。

\subsection{3 个模型拟合 3 种植物的 $\Phi_{\mathrm{PSIImax}} 、 F_{\mathrm{v}} / F_{\mathrm{m}}$ 和 $\alpha / \omega$ 的结果比较}

由(8)和(9)式可以估算植物的 $\Phi_{\mathrm{PSIImax}}$, 由(10)和 (11)式可以估算植物的 $F_{\mathrm{v}} / F_{\mathrm{m}}$, 但对比表 1 和表 2 中的 数据可知, 由(8)和(9)式估算同种植物的 $\Phi_{\mathrm{PSIImax}}$ 得 到的值并不相同，由(10)和(11)式估算同种植物的 

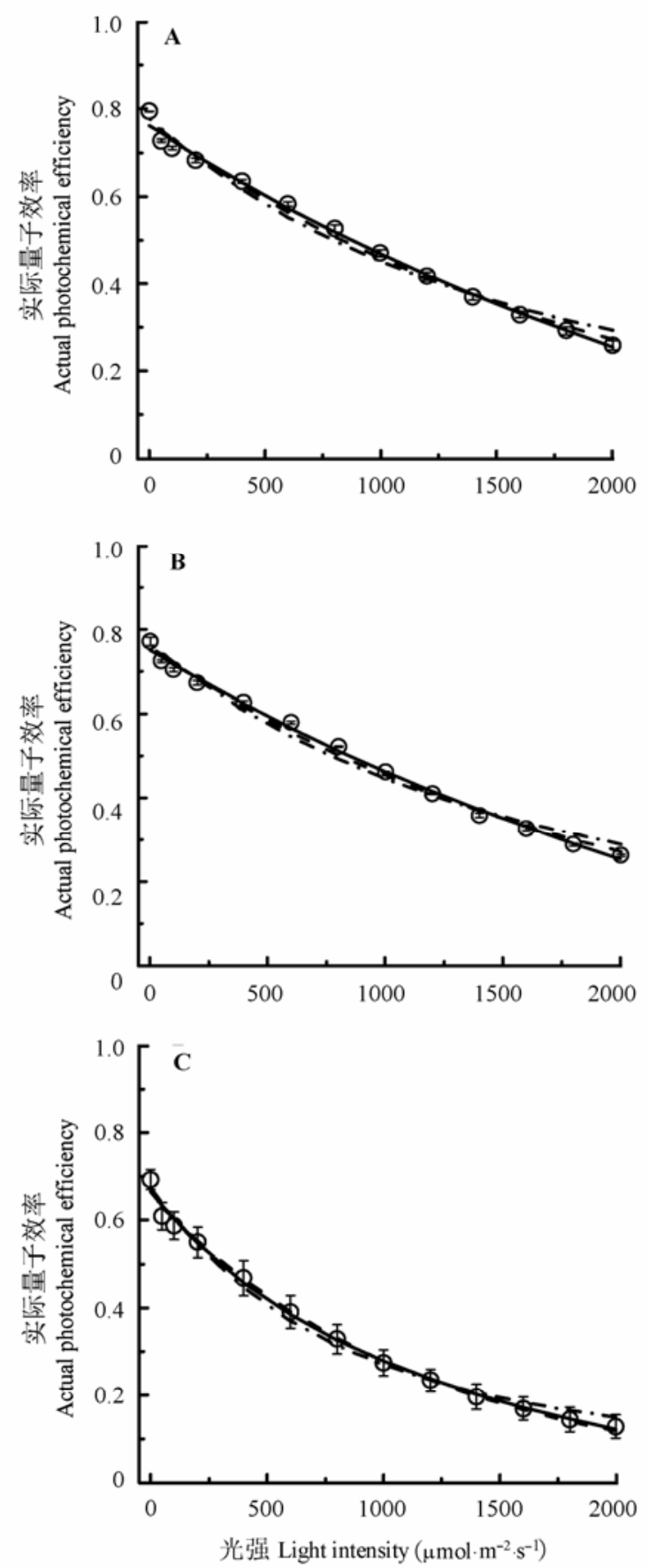

○ 测量点 Measured point - - 公式(8) Equation (8) 公式(5) Equation (5) - - - 公式(10) Equation (10)

图2 剑叶金鸡菊 $(A)$ 、黄荆 $(B)$ 和大狼杷草 $(\mathbf{C})$ 的实际光量子 效率对光的响应曲线 $\left(\Phi_{\mathrm{PSII}}-I\right)$ (平均值 \pm 标准误差)。公式(5)为 $\Phi_{\mathrm{PSII}}=\omega(1-\beta I) /(1+\gamma I)$, 其中 $\omega$ 为 $\Phi_{\mathrm{PSII}}-I$ 的初始斜率; 公式 (8)为 $\Phi_{\mathrm{PSII}}=\Phi_{\mathrm{PSIImax}} \mathrm{e}^{-k_{\mathrm{w}} I}$, 公式(10)为 $\Phi_{\mathrm{PSII}}=\left(F_{\mathrm{v}} / F_{\mathrm{m}}\right) P A R_{\mathrm{sat}} / I$ $\left(1-\exp \left(-I / P A R_{\mathrm{sat}}\right)\right) 。 \beta 、 \gamma 、 F_{\mathrm{v}} / F_{\mathrm{m}} 、 \Phi_{\mathrm{PSIImax}} 、 k_{\mathrm{w}}$ 和 $P A R_{\mathrm{sat}}$ 的定 义同图1。

Fig. 2 Light-response curves of $\Phi_{\mathrm{PSII}}$ for Coreopsis lanceolata (A), Vitex negundo (B) and Bideas frondosa $(\mathbf{C})($ mean $\pm S E)$. Equation (5) is $\Phi_{\text {PSII }}=\omega(1-\beta I) /(1+\gamma I)$. Here $\omega$ is the initial slope of $\Phi_{\text {PSII }} I$; Equation (8) is $\Phi_{\text {PSII }}=\Phi_{\text {PSIImax }} \mathrm{e}^{-k_{w} I}$; Equation (10) is $\Phi_{\text {PSII }}=\left(F_{\mathrm{v}} / F_{\mathrm{m}}\right) P A R_{\text {sat }} / I\left(1-\exp \left(-I / P A R_{\text {sat }}\right)\right)$. The definition of $\beta, \gamma, F_{\mathrm{v}} / F_{\mathrm{m}}, \Phi_{\mathrm{PSIImax}}, k_{\mathrm{w}}$ and $P A R_{\text {sat }}$ see Fig. 1.
$F_{\mathrm{v}} / F_{\mathrm{m}}$ 得到的值也不相同。例如，用(9)式得到剑叶金 鸡菊的 $\Phi_{\mathrm{PSIImax}}$ 为 0.859 , 而由(8)式得到剑叶金鸡菊 的 $\Phi_{\mathrm{PSIImax}}$ 为 0.771 , 前者是后者的 1.114 倍。为了确定 这些模型估算的 $\Phi_{\mathrm{PSIImax}}$ 之间和 $F_{\mathrm{v}} / F_{\mathrm{m}}$ 之间是否存在 差异, 对拟合结果进行了ANOVA检验, 发现用(8) 和(9)式分别得到的剑叶金鸡菊和黄荆的 $\Phi_{\mathrm{PSII} \text { max }}$ 值 之间存在显著差异(表3); 同时也对由(10)和(11)式 估算的植物 $F_{\mathrm{v}} / F_{\mathrm{m}}$ 的值进行了ANOVA检验, 发现用 (10)和(11)式分别得到的剑叶金鸡菊(和黄荆)的 $F_{\mathrm{v}} / F_{\mathrm{m}}$ 值之间也存在显著差异(表3)。此外, 由配对样 本 $t$ 检验得知: 由(2)式和(9)式或(5)式和(8)式得到3 种植物的 $P A R_{\mathrm{sat}}$ 与实测值之间不存在显著差异, 而 与(10)或(11)式得到3种植物的 $P A R_{\mathrm{sat}}$ 与实测值之间 则存在显著差异(数据没有给出)。

此外, 对 $\alpha / \omega$ 和0.42 $\left(\alpha^{\prime} \beta^{\prime}=0.42\right)$ 之间进行了配 对样本 $t$ 检验, 发现剑叶金鸡菊和黄荆的 $\alpha / \omega$ 和 0.42 存在显著差异(表3)。由此可知剑叶金鸡菊、黄荆和 大狼杷草的 $\alpha / \omega$ 值分别为 $0.455 、 0.468$ 和 0.474 , 这 3 个值都比LI-6400-40B给出 $\alpha^{\prime} \beta^{\prime}$ 的推荐值 0.42 大。

\section{4 三种植物天线色素分子的有效光能吸收截面} 对光的响应曲线 $\left(\sigma^{\prime}{ }^{\prime}-I\right)$

天线色素分子的有效光能吸收截面 $\left(\sigma_{\mathrm{ik}}^{\prime}\right)$ 表示其 在不同光强下天线色素分子捕获光子能力的大小。 由图3可知, 剑叶金鸡菊、黄荆和大狼杷草的天线色 素分子有效光能吸收截面随光强的增加而下降, 表 明这 3 种植物随光强的增加其捕获光子的能力都在 逐渐变小。由图 3 和表 1 的数据可知, 剑叶金鸡菊和 黄荆天线色素分子在高光强 $\left(2000 \mu \mathrm{mol} \cdot \mathrm{m}^{-2} \cdot \mathrm{s}^{-1}\right)$ 时, 它们的有效光能吸收截面几乎相同, 约为 $1.85 \times$ $10^{-21} \mathrm{~m}^{2}$, 表明这两种植物在高光强时依然可以吸 收较多的光能。而大狼杷草天线色素分子的有效光 能吸收截面在高光强 $\left(2000 \mu \mathrm{mol} \cdot \mathrm{m}^{-2} \cdot \mathrm{s}^{-1}\right)$ 时约为 $1.02 \times 10^{-21} \mathrm{~m}^{2}$, 表明在高光强时, 它吸收的光能比 剑叶金鸡菊和黄荆少 $81 \%$ 左右。

此外, 对比图3和图2可知, 剑叶金鸡菊、黄荆和 大狼杷草的 $\Phi_{\mathrm{PSII}}-I$ 曲线与它们的 $\sigma_{\mathrm{ik}}^{\prime}-I$ 曲线非常相似。

\section{3 讨论和结论}

首先, 基于光合电子流对光响应的机理模型构 建了一个 $\Phi_{\mathrm{PSII}}$ 对光的响应模型。由 $\Phi_{\mathrm{PSII}}-\mathrm{I}$ 模型可知, $\Phi_{\mathrm{PSII}}$ 不仅与光强有关, 而且还与植物天线色素的内 禀特性存在非线性的关系, 即 $\Phi_{\mathrm{PSII}}$ 的大小除了与叶 
表1 三种模型对三种植物光合电子流对光曲线的拟合参数

Table 1 The measured data and fitted values for Coreopsis lanceolata, Vitex negundo and Bidens frondosa using three models

\begin{tabular}{|c|c|c|c|c|c|c|c|c|c|c|c|c|}
\hline \multirow{2}{*}{$\begin{array}{l}\text { 光合参数 } \\
\text { Photosynthetic } \\
\text { parameter }\end{array}$} & \multicolumn{4}{|c|}{ 剑叶金鸡菊 C. lanceolata } & \multicolumn{4}{|c|}{ 黄荆 V. negundo } & \multicolumn{4}{|c|}{ 大狼杷草 B. frondosa } \\
\hline & $\begin{array}{l}\text { 公式(2) } \\
\text { Equation } \\
(2)\end{array}$ & $\begin{array}{l}\text { 公式(9) } \\
\text { Equation } \\
(9) \\
\end{array}$ & $\begin{array}{l}\text { 公式(11) } \\
\text { Equation } \\
(11) \\
\end{array}$ & $\begin{array}{l}\text { 测量值 } \\
\text { Measured } \\
\text { value } \\
\end{array}$ & $\begin{array}{l}\text { 公式(2) } \\
\text { Equation } \\
(2)\end{array}$ & $\begin{array}{l}\text { 公式(9) } \\
\text { Equation } \\
(9)\end{array}$ & $\begin{array}{l}\text { 公式(11) } \\
\text { Equation } \\
(11) \\
\end{array}$ & $\begin{array}{l}\text { 测量值 } \\
\text { Measured } \\
\text { value } \\
\end{array}$ & $\begin{array}{l}\text { 公式(2) } \\
\text { Equation } \\
(2)\end{array}$ & $\begin{array}{l}\text { 公式(9) } \\
\text { Equation } \\
(9) \\
\end{array}$ & $\begin{array}{l}\text { 公式(11) } \\
\text { Equation } \\
(11) \\
\end{array}$ & $\begin{array}{l}\text { 测量值 } \\
\text { Measured } \\
\text { value } \\
\end{array}$ \\
\hline $\begin{array}{l}\text { ETR-I的初始斜率 } \\
\text { Initial slope of ETR-I } \\
\left(\mu \mathrm{mol} \cdot \mu \mathrm{mol}^{-1}\right)\end{array}$ & 0.347 & $\begin{array}{c}\Phi_{\text {PSIImax }} \\
(0.859)\end{array}$ & $\begin{array}{l}F_{\mathrm{v}} / F_{\mathrm{m}} \\
(0.954)\end{array}$ & - & 0.352 & $\begin{array}{c}\Phi_{\text {PSIImax }} \\
(0.834)\end{array}$ & $\begin{array}{l}F_{\mathrm{v}} / F_{\mathrm{m}} \\
(0.928)\end{array}$ & - & 0.315 & $\begin{array}{l}\Phi_{\text {PSIImax }} \\
(0.675)\end{array}$ & $\begin{array}{l}F_{\mathrm{v}} / F_{\mathrm{m}} \\
(0.820)\end{array}$ & - \\
\hline $\begin{array}{l}\text { 最大电子传递速率 } \\
\text { Maximu electron } \\
\text { transport rate } \\
\left(\mu \mathrm{mol} \cdot \mathrm{m}^{-2} \cdot \mathrm{s}^{-1}\right)\end{array}$ & 232.3 & 231.3 & 156.5 & 230.4 & 230.2 & 229.8 & 155.99 & $\approx 228.8$ & 136.7 & 137.28 & 86.67 & $\approx 135.8$ \\
\hline $\begin{array}{l}\text { 饱和光强 } \\
\text { Saturation irradiance } \\
\left(\mu \mathrm{mol} \cdot \mathrm{m}^{-2} \cdot \mathrm{s}^{-1}\right)\end{array}$ & 1641.7 & 1742.2 & 617.83 & $\approx 1600$ & 1740.9 & 1782.5 & 633.1 & $\approx 1750$ & 1394.5 & 1326.3 & 395.2 & $\approx 1400$ \\
\hline $\begin{array}{l}\text { 叶绿素含量 } \\
\text { Chlorophyll content } \\
\left(\mathrm{mg} \cdot \mathrm{m}^{-2}\right)\end{array}$ & 253.68 & & & & 268.18 & & & & 286.33 & & & \\
\hline $\begin{array}{l}\text { 本征截面 } \\
\text { Eign-absorption cross } \\
\text { section } \\
\left(10^{-21} \mathrm{~m}^{2}\right)\end{array}$ & 5.41 & - & - & - & 5.19 & - & - & - & 4.35 & - & - & - \\
\hline $\begin{array}{l}\text { 决定系数 } \\
\text { Determined coefficient }\end{array}$ & 1.000 & 0.998 & 0.995 & - & 0.999 & 0.999 & 0.997 & - & 0.998 & 0.997 & 0.995 & - \\
\hline $\begin{array}{l}\text { AIC信息准则 } \\
\text { Akaike’s information } \\
\text { criterion }\end{array}$ & 7.40 & 7.34 & 9.22 & - & 8.34 & 7.48 & 8.78 & - & 7.74 & 7.27 & 8.46 & - \\
\hline
\end{tabular}

公式(2)、(9)、(11)同图1。

Equation (2), Equation (9), Equation (11) see Fig. 1.

表2 三种模型对三种植物叶片的 $\Phi_{\mathrm{PSII}}-I$ 曲线的拟合参数

Table 2 The measured data and fitted values for Coreopsis lanceolata, Vitex negundo and Bidens frondosa using three models

\begin{tabular}{|c|c|c|c|c|c|c|c|c|c|c|c|c|}
\hline \multirow{2}{*}{$\begin{array}{l}\text { 光合参数 } \\
\text { Photosynthetic } \\
\text { parameter }\end{array}$} & \multicolumn{4}{|c|}{ 剑叶金鸡菊 C. lanceolata } & \multicolumn{4}{|c|}{ 黄荆 V. negundo } & \multicolumn{4}{|c|}{ 大狼杷草 B. frondosa } \\
\hline & $\begin{array}{l}\text { 公式(5) } \\
\text { Equation } \\
(5)\end{array}$ & $\begin{array}{l}\text { 公式(8) } \\
\text { Equation } \\
(8) \\
\end{array}$ & $\begin{array}{l}\text { 公式(10) } \\
\text { Equation } \\
(10)\end{array}$ & $\begin{array}{l}\text { 测量值 } \\
\text { Measured } \\
\text { value }\end{array}$ & $\begin{array}{l}\text { 公式(5) } \\
\text { Equation } \\
\text { (5) }\end{array}$ & $\begin{array}{l}\text { 公式(8) } \\
\text { Equation } \\
\text { (8) }\end{array}$ & $\begin{array}{l}\text { 公式(10) } \\
\text { Equation } \\
(10)\end{array}$ & $\begin{array}{l}\text { 测量值 } \\
\text { Measured } \\
\text { value }\end{array}$ & $\begin{array}{l}\text { 公式(5) } \\
\text { Equation } \\
(5)\end{array}$ & $\begin{array}{l}\text { 公式(8) } \\
\text { Equation } \\
(8) \\
\end{array}$ & $\begin{array}{l}\text { 公式(10) } \\
\text { Equation } \\
(10)\end{array}$ & $\begin{array}{l}\text { 测量值 } \\
\text { Measured } \\
\text { value }\end{array}$ \\
\hline $\begin{array}{l}\Phi_{\mathrm{PSII}}-I \text { 的初始斜率 } \\
\text { Initial slope of } \Phi_{\mathrm{PSII}}-I \\
\left(\mu \mathrm{mol} \cdot \mu \mathrm{mol}^{-1}\right)\end{array}$ & 0.763 & $\begin{array}{l}\Phi_{\text {PSIImax }} \\
(0.771)\end{array}$ & $\begin{array}{l}F_{\mathrm{v}} / F_{\mathrm{m}} \\
(0.779)\end{array}$ & - & 0.752 & $\begin{array}{l}\Phi_{\text {PSIImax }} \\
(0.759)\end{array}$ & $\begin{array}{l}F_{\mathrm{v}} / F_{\mathrm{m}} \\
(0.767)\end{array}$ & - & 0.665 & $\begin{array}{l}\Phi_{\text {PSIImax }} \\
(0.678)\end{array}$ & $\begin{array}{l}F_{\mathrm{v}} / F_{\mathrm{m}} \\
(0.674)\end{array}$ & - \\
\hline $\begin{array}{l}\text { 饱和光强 } \\
\text { Saturation irradiance } \\
\left(\mu \mathrm{mol} \cdot \mathrm{m}^{-2} \cdot \mathrm{s}^{-1}\right)\end{array}$ & 1642.9 & 1744.9 & 824.95 & $\approx 1600$ & 1712.7 & 1763.3 & 830.33 & $\approx 1750$ & 1406.7 & 1331.2 & 448.50 & $\approx 1400$ \\
\hline $\begin{array}{l}\text { 决定系数 } \\
\text { Determined coefficient }\end{array}$ & 0.995 & 0.992 & 0.984 & - & 0.996 & 0.994 & 0.986 & - & 0.996 & 0.995 & 0.991 & - \\
\hline $\begin{array}{l}\text { AIC信息准则 } \\
\text { Akaike’s information } \\
\text { criterion }\end{array}$ & -2.49 & -2.75 & -2.03 & - & -2.79 & -3.04 & -2.20 & - & -2.51 & -3.06 & -2.46 & - \\
\hline
\end{tabular}

公式(5)、(8)、(10)同图2。

Equation (5), Equation (8), Equation (10) see Fig. 2.

表3 三种模型对三种植物叶片的 $\Phi_{\mathrm{PSII}}-I$ 曲线的拟合参数(平均值土标准误差)

Table 3 The fitted values of $\Phi_{\mathrm{PSII}}-I$ curves for Coreopsis lanceolata, Vitex negundo and Bidens frondosa using three models (mean \pm SE)

\begin{tabular}{|c|c|c|c|c|c|c|}
\hline & \multicolumn{2}{|c|}{ 剑叶金鸡菊 C. lanceolata } & \multicolumn{2}{|c|}{ 黄荆 V. negundo } & \multicolumn{2}{|c|}{ 大狼杷草 B. frondosa } \\
\hline & $\Phi_{\text {PSIImax }}$ & $F_{\mathrm{v}} / F_{\mathrm{m}}$ & $\Phi_{\text {PSIImax }}$ & $F_{\mathrm{v}} / F_{\mathrm{m}}$ & $\Phi_{\text {PSIImax }}$ & $F_{\mathrm{v}} / F_{\mathrm{m}}$ \\
\hline 公式(8) Equation (8) & $0.859 \pm 0.011^{\mathrm{a}}$ & - & $0.834 \pm 0.005^{\mathrm{a}}$ & - & $0.678 \pm 0.059^{\mathrm{a}}$ & - \\
\hline 公式(9) Equation (9) & $0.771 \pm 0.004^{\mathrm{b}}$ & - & $0.759 \pm 0.006^{\mathrm{b}}$ & - & $0.675 \pm 0.051^{\mathrm{a}}$ & - \\
\hline 公式(10) Equation (10) & - & $0.954 \pm 0.019^{\mathrm{a}}$ & - & $0.928 \pm 0.005^{\mathrm{a}}$ & - & $0.820 \pm 0.079^{\mathrm{a}}$ \\
\hline 公式(11) Equation (11) & - & $0.779 \pm 0.004^{\mathrm{b}}$ & - & $0.767 \pm 0.006^{\mathrm{b}}$ & - & $0.674 \pm 0.029^{\mathrm{a}}$ \\
\hline$\alpha / \omega$ & \multicolumn{2}{|c|}{$0.455 \pm 0.007^{*}$} & \multicolumn{2}{|c|}{$0.468 \pm 0.008^{*}$} & \multicolumn{2}{|c|}{$0.474 \pm 0.023^{\mathrm{ns}}$} \\
\hline
\end{tabular}

公式(8)、(9)、(10)、(11)同图1和图2。不同小写字母 $\left(\mathrm{a}\right.$ 和b)表示同一参数在相同模型间存在显著性差异 $(p<0.05) 。 *, \alpha / \omega$ 和 $0.42\left(\alpha^{\prime} \beta^{\prime}=0.42\right)$ 之间存在显 著性差异 $(p<0.05)$ 。

Equation (8), Equation (9), Equation (10), Equation (11) see Fig. 1 and Fig. 2. Different letters (a and b) indicate statistical significance at $p<0.05$ between the same model. *, significant difference $(p<0.05)$ between $\alpha / \omega$ and $0.42\left(\alpha^{\prime} \beta^{\prime}=0.42\right)$. 


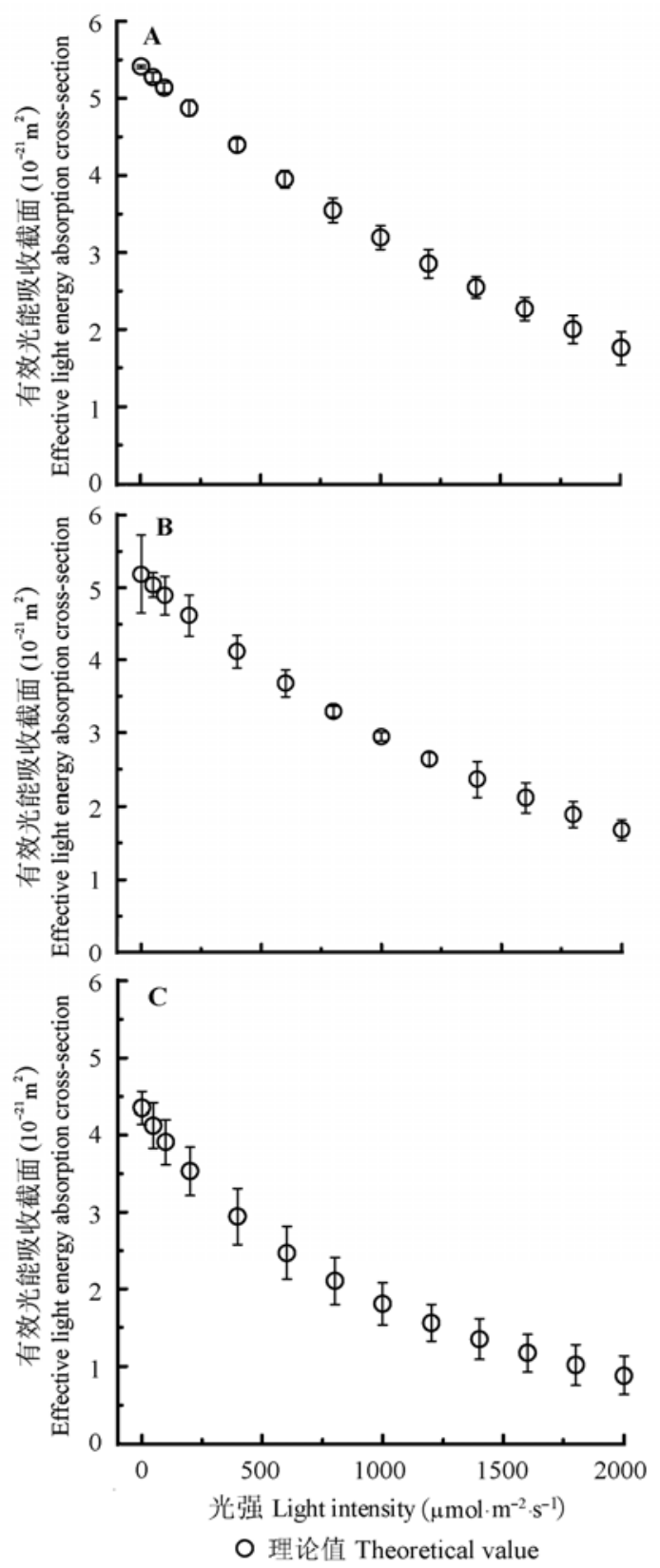

图3 剑叶金鸡菊 $(\mathbf{A})$ 、黄荆 $(\mathbf{B})$ 和大狼杷草 $(\mathbf{C})$ 的有效光能吸 收截面对光的响应曲线 $\left(\sigma_{\mathrm{ik}}^{\prime}-I\right)$ (平均值土标准误差)。

Fig. 3 Light-response curves of effective light energy absorption cross-section $\left(\sigma_{\mathrm{ik}}^{\prime}-I\right)$ for Coreopsis lanceolata (A), Vitex negundo $(\mathbf{B})$ and Bidens frondosa $(\mathbf{C})($ mean $\pm S E)$.

绿素的含量有关外, 还与天线色素分子的本征光能 吸收截面和激子的传递效率等有关。

其次, 指数模型和负指数模型主要用于藻类 (Laws et al., 2002; Ritchie, 2008)、浮游植物(Smyth et al., 2004; Silsbe \& Kromkamp, 2012)和少数几种陆 生植物(Ritchie, 2008; Ritchie \& Bunthawin, 2010), 用于陆地高等植物较少。本研究中, 我们分别用所 构建的 $\Phi_{\mathrm{PSII}}$ 对光响应的机理模型, 同时用负指数模
型和指数模型分别拟合了剑叶金鸡菊、黄荆和大狼 杷草的 $\Phi_{\mathrm{PSII}}-{ }$ 响应曲线, 发现这3个模型都可以较好 地拟合这3种植物的 $\Phi_{\mathrm{PSII}}-I$ 响应曲线, $R^{2}$ 最低为 0.984 , 但由指数模型拟合得到这 3 种植物的 $P A R_{\mathrm{sat}}$ 与 实测值相差较大，约为实测值的一半甚至更少(表 2 )。由配对样本 $t$ 检验也可以得知: 由公式(10)或(11) 得到 3 种植物的 $P A R_{\mathrm{sat}}$ 与实测值之间存在显著差异, 而由公式(3)和(5)或公式(8)和(9)得到3种植物的 $P A R_{\mathrm{sat}}$ 与实测值之间不存在显著差异(数据并没有给 出), 这表明由机理模型和指数模型拟合得到的 $P A R_{\text {sat }}$ 与实测值相符。此外, 我们用这 3 个 $\Phi_{\mathrm{PSII}}-I$ 响应 模型所对应的ETR-I响应模型分别拟合了剑叶金鸡 菊、黄荆和大狼杷草的ETR-I响应曲线。如果仅用 $R^{2}$ 来判断 3 个模型拟合的优劣, 那么以 $E T R-I$ 和 $\Phi_{\mathrm{PSII}}-\mathrm{I}$ 机理模型为最佳, 指数模型最差; 如果以 AIC 作为判断标准, 则以负指数模型为最佳, 指数模型 为最差。而由图1、图2、表1和表2的拟合结果可知, 只有 $E T R-I$ 的机理模型和负指数 ETR-I的响应模型拟 合得到的 $E T R_{\mathrm{max}}$ 和 $P A R_{\mathrm{sat}}$ 与实测值高度符合, 而由 指数 $E T R-I$ 的响应模型得到的 $E T R_{\mathrm{max}}$ 和 $P A R_{\mathrm{sat}}$ 远低于 实测值。这些研究结果与前人的结果相似(Laws et al., 2002; Smyth et al., 2004; Silsbe \& Kromkamp, 2012)。由此可知, 与机理模型和负指数模型的拟合 结果相比, 指数模型不适于研究藻类、浮游植物和 陆地植物的 $\Phi_{\mathrm{PSII}}-I$ 响应和ETR-I响应问题。

再次, 大量的实验数据表明: $\Phi_{\mathrm{PSII}}$ 随光强的增 加而下降是一个普遍现象(Ritchie, 2008; Ritchie \& Bunthawin, 2010; Silsbe \& Kromkamp, 2012; 冯汉青 等, 2014; 安东升等, 2015; 林琭等, 2015; 师生波 等, 2015; Ware et al., 2015)。本研究中剑叶金鸡菊、 黄荆和大狼杷草的 $\Phi_{\mathrm{PSII}}$ 也随光强的增加而下降(图 2), 与前人的结果相似。此外, 由图1和图2可知, 负 指数模型和指数模型可以拟合三种植物的 ETR-I和 $\Phi_{\mathrm{PSII}}-I$ 响应曲线, 却不能解释 $\Phi_{\mathrm{PSII}}$ 随光强的增加而 下降的原因, 对应的电子传递速率对光的响应模型 即公式(9)和(11)也不能解释在ETR-I响应曲线中出 现的光系统II的动力学下调 (White \& Critchley, 1999; Ralph \& Gademann, 2008)或光抑制(Vijayan \& Browse, 2002; Goh et al., 2012; Rochaix, 2014; Murchie et al., 2015)的原因。然而由公式(7) $\Phi_{\mathrm{PSII}}$ 与 $\sigma_{\mathrm{ik}}{ }^{\prime}$ 之间的关系可知, 剑叶金鸡菊、黄荆和大狼杷草 的 $\Phi_{\mathrm{PSII}}$ 随光强的增加而非线性下降的原因来自它们 
的天线色素分子的有效光能吸收截面随光强的增加 而下降(图3), 即天线色素分子吸收光子的能力随光 强的增加而减少, 由此 3 种植物用于光合作用的光 能就减少了, 从而导致PSII的实际光化学量子效率 降低。

最后，用公式(8)和(9)分别得到的剑叶金鸡菊 和黄荆的 $\Phi_{\mathrm{PSII} m a x}$ 值之间存在显著差异(表3); 用公 式(10)和(11)估算得到剑叶金鸡菊和黄荆的 $F_{\mathrm{v}} / F_{\mathrm{m}}$ 值 之间也存在显著差异(表3)。此外, 剑叶金鸡菊、黄 荆和大狼杷草的 $\alpha / \omega$ 值分别为 $0.455 、 0.468$ 和 0.474 , 其中剑叶金鸡菊和黄荆 $\alpha / \omega$ 的值与 $0.42\left(\alpha^{\prime} \beta^{\prime}=0.42\right)$ 存在显著差异(表3), 也即这3个值都比LI- 6400-40B 给出 $\alpha^{\prime} \beta^{\prime}$ 的推荐值 0.42 要大。其原因可能是剑叶金鸡 菊、黄荆和大狼杷草叶片的光能吸收系数 $\left(\beta^{\prime}\right)$ 要大于 0.84 或光能分配到 PSII的系数要大于 0.5 , 也即由 LI-6400-40B给出 $\alpha^{\prime}$ 和 $\beta^{\prime}$ 的推荐值不适合这3种植物。 如果能用球面积分仪测量植物叶片的光吸收系数 $\left(\beta^{\prime}\right)$, 就可以由公式(2)和(5)或由公式(8)和(9)计算叶 片吸收的光能是否平均地分配到光系统II和光系统 $I$, 即可以确定 $\alpha^{\prime}$ 是否恒等于 0.5 。这为研究植物光能 是否平均分配到光系统II和光系统I提供了一个有效 工具。

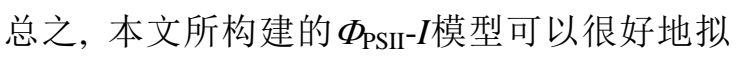
合3种植物PSII的实际光化学量子效率对光的响应 曲线, 并可以很好地说明 $\Phi_{\mathrm{PSII}}$ 随光强的增加而非线 性下降的原因是捕光色素分子的有效光能吸收截面 随光强的增加而下降。同时, 其对应的ETR-I响应模 型可以拟合不同植物的电子传递速率对光的响应曲 线, 并可以合理地解释植物的PSII动力学下调或光 抑制等生理现象。此外, 当 $\Phi_{\mathrm{PSII}}-I$ 模型和ETR-I机理 模型相结合, 并结合球面积分仪测量植物叶片的光 吸收系数时, 就可以研究植物叶片吸收的光能是否 平均地分配到PSII和PSI。

基金项目 国家自然科学基金(31560069)和江西省 自然科学基金(20142BAB20402)。

\section{参考文献}

Akaike H (1973). A new look at the statistical model identification. IEEE Translations Automatic Control, 19, 716-723.

An DS, Cao J, Huang XH, Zhou J, Dou MA (2015). Application of Lake-model based indices from chlorophyll fluorescence on sugarcane seedling drought resistance study. Chinese Journal of Plant Ecology, 39, 398-406. (in Chi- nese with English abstract) [安东升, 曹娟, 黄小华, 周 娟, 窦美安 (2015). 基于Lake模型的叶绿素荧光参数在 甘蔗苗期抗旱性研究中的应用. 植物生态学报, 39, 398-406.]

Arnon DJ (1949). Copper enzymes in isolated chloroplasts. Polyphenoloxidase in Beta vulgaris. Plant Physiology, 24, 1-15.

Baker NR (2008). Chlorophyll fluorescence: A probe of photosynthesis in vivo. Annual of Review Plant Biology, 59, 89-113.

Ehleringer J, Pearcy RW (1983). Variation in quantum yield for $\mathrm{CO}_{2}$ uptake among $\mathrm{C}_{3}$ and $\mathrm{C}_{4}$ plants. Plant Physiology, 73, 555-559.

Feng HQ, Jiao QS, Tian WY, Sun K, Jia LY (2014). Effects of extracellular ATP on the characteristics of photochemical reaction in bean (Phaseolus vulgaris) leaves under different light intensities. Chinese Journal of Plant Ecology, 38, 1117-1123. (in Chinese with English abstract) [冯汉青, 焦青松, 田武英, 孙坤, 贾凌云 (2014). 不同光强下细 胞外三磷酸腺苷对菜豆叶片光化学反应特性的影响. 植物生态学报, 38, 1117-1123.]

Genty B, Briantais JM, Baker NR (1989). The relationship between the quantum yield of photosynthetic electron transport and quenching of chlorophyll fluorescence. Biochimica et Biophysica Acta, 990, 87-92.

Goh CH, Ko SM, Koh S, Kim YJ, Bae HJ (2012). Photosynthesis and environments: Photoinhibition and repair mechanisms in plants. Journal of Plant Biology, 55, 93-101.

Govindjee (2002). A role for a light-harvesting antenna complex of photosystem II in photoprotection. The Plant Cell, 14, 1663-1668.

Krall JP, Edward GE (1992). Relationship between photosystem II activity and $\mathrm{CO}_{2}$ fixation in leaves. Physiologia Plantarum, 86,180-187.

Laws E, Sakshaug E, Babin M, Dandonneau Y, Falkowski P, Geider R, Legendre L, Morel A, Sondergaard M, Takahashi M, Williams PJ (2002). Photosynthesis and primary productivity in marine ecosystems: Practical aspects and application of techniques. Joint Global Ocean Flux Study, 36, 1-77.

Lin L, Tang Y, Zhang JT, Yan WL, Xiao JH, Ding C, Dong C, Ji ZS (2015). Effects of different water potentials on leaf gas exchange and chlorophyll fluorescence parameters of cucumber during post-flowering growth stage. Chinese Journal of Applied Ecology, 26, 2030-2040. (in Chinese with English abstract) [林琭, 汤昀, 张纪涛, 间万丽, 肖 建红, 丁超, 董川, 籍增顺 (2015). 不同水势对黄瓜花 后叶片气体交换及叶绿素荧光参数的影响. 应用生态 学报, 26, 2030-2040.]

Lin MZ, Wang ZW, He LC, Xu K, Cheng DL, Wang GX (2015). Plant photosynthesis-irradiance curve response to 
pollution show non-competitive inhibited Michaelis kinetics. PLOS ONE, 10, e0142712. doi: 10.1371/journal. pone. 0142712.

Major KM, Dunton KH (2002). Variations in light-harvesting characteristics of the seagrass, Thalassia testudinum: Evidence for photoacclimation. Journal of Experimental Marine Biology and Ecology, 275, 173-189.

Murchie EH, Ali A, Herman T (2015). Photoprotection as a trait for rice yield improvement: Status and prospects. Rice, 8, 31-40.

Nelson N, Yocum CF (2006). Structure of function of photosystems I and II. Annual Review of Plant Biology, 57, 521-565.

Niyogi KK, Truong TB (2013). Evolution of flexible nonphotochemical quenching mechanisms that regulate light harvesting in oxygenic photosynthesis. Current Opinion in Plant Biology, 16, 307-314.

Oxborough K (2004). Imaging of chlorophyll a fluorescence: Theoretical and practical aspects of an emerging technique for the monitoring of photosynthetic performance. Journal of Experimental Botany, 55, 1195-1205.

Panitchayangkoon G, Hayes D, Fransted KA, Caram JR, Harel E, Wen J, Blankenship RE, Engel GS (2010). Long-lived quantum coherence in photosynthetic complexes at physiological temperature. Proceedings of the National Academy Sciences of the United States of America, 107, 12766-12770.

Ralph PJ, Gademann R (2005). Rapid light curves: A powerful tool to assess photosynthetic activity. Aquatic Botany, 82, 222-237.

Richter M, Renger T, Knorr A (2008). A Block equation approach to intensity dependent optical spectra of light harvesting complex II. Excitation dependence of light harvesting complex II pump-probe spectra. Photosynthesis Research, 95, 119-127.

Ritchie RJ (2008). Fitting light saturation curves measured using modulated fluorometry. Photosynthesis Research, 96, 201-215.

Ritchie RJ, Bunthawin S (2010). The use of pulse amplitude modulation (PAM) fluorometry to measure photosynthesis in a cam orchid, Dendrobium spp. (D. cv. Viravuth Pink). International Journal of Plant Sciences, 171, 575-585.

Rochaix JD (2014). Regulation and dynamics of the lightharvesting system. Annual of Review Plant Biology, 65, 287-309.

Sarovar M, Ishizaki A, Fleming G, Whaley B (2010). Quantum entanglement in photosynthetic light-harvesting complexes. Nature Physics, 6, 462-467.

Shi SB, Li TC, Li M, Liu SZ, Li AD, Ma JP (2015). Interaction effect analysis of soil drought and strong light on PSII nonphotochemical quenching in Kobresia pygmaea leaves. Plant Physiology Journal, 51, 1678-1686. (in Chinese with English abstract) [师生波, 李天才, 李妙, 刘世增, 李爱德，马剑平 (2015). 土壤干旱和强光对高山嵩草叶 片PSII反应中心非光化学猝灭的交互影响分析. 植物生 理学报, 51, 1678-1686.]

Silsbe GM, Kromkamp JC (2012). Modeling the irradiance dependency of the quantum efficiency of photosynthesis. Limnology and Oceanography: Methods, 10, 645-652.

Smyth TJ, Pemberton KL, Aiken J, Geider RJ (2004). A methodology to determine primary production and phytoplankton photosynthetic parameters from fast repetition rate fluorometry. Journal of Plankton Research, 26, 13371350.

Takahashi S, Badger MR (2011). Photoprotection in plants: A new light on photosystem II damage. Trends in Plant Sciences, 16, 53-59.

Vijayan P, Browse J (2002). Photoinhibition in mutants of arabidopsis deficient in thylakoid unsaturation. Plant Physiology, 129, 876-885.

Ware MA, Belgio E, Ruban AV (2015). Photoprotective capacity of non-photochemical quenching in plants acclimated to different light intensities. Photosynthesis Research, 126, 261-274.

Webb WL, Newton M, Starr D (1974). Carbon dioxide exchange of Alnus rubra: A mathematical model. Oecologia, 17, 281-291.

White AJ, Critchley C (1999). Rapid light curves: A new fluorescence method to asses the state of the photosynthetic apparatus. Photosynthesis Research, 59, 63-72.

Ye ZP, Hu WH, Xiao YA, Fan DY, Yin JH, Duan SH, Yan XH, He L, Zhang SS (2014). A mechanistic model of light-response of photosynthetic electron flow and its application. Chinese Journal of Plant Ecology, 38, 12411249. (in Chinese with English abstract) [叶子飘, 胡文海, 肖宜安, 㚞大勇, 尹建华, 段世华, 间小红, 贺俐, 张斯 斯 (2014). 光合电子流对光响应的机理模型及其应用. 植物生态学报, 38, 1241-1249.]

Ye ZP, Robakowski P, Suggett DJ (2013a). A mechanistic model for the light response of photosynthetic electron transport rate based on light harvesting properties of photosynthetic pigment molecules. Planta, 237, 837-847.

Ye ZP, Suggett DJ, Robakowski P, Kang HJ (2013b). A mechanistic model for the photosynthesis-light response based on the photosynthetic electron transport of PSII in $\mathrm{C}_{3}$ and $\mathrm{C}_{4}$ species. New Phytologist, 199, 110-120.

责任编委: 王根轩 责任编辑: 李 敏 


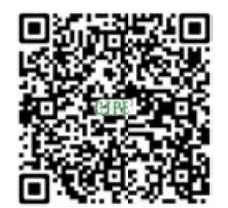

植物生态学报官网

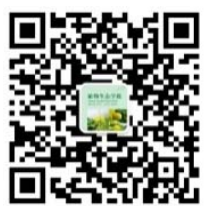

微信订阅号

期刊及学科

相关信息发布

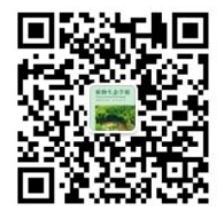

微信服务号

稿件状态查询

全文检索汶览 